\title{
Irradiation Induced Tunnel Barrier in Side-gated Graphene Nanoribbon
}

\author{
Shuojin Hang ${ }^{1}$, Zakaria Moktadir ${ }^{1}$, and Hiroshi Mizuta ${ }^{1,2}$ \\ ${ }^{1}$ Faculty of Physical Sciences and Engineering, University of Southampton, UK \\ ${ }^{2}$ School of Materials Science, Japan Advanced Institute of Science and Technology (JAIST), Japan \\ Email: sh13g08@ecs.soton.ac.uk
}

\begin{abstract}
We investigated a method of forming tunnel barriers in monolayer graphene nanoribbon (GNR) using controlled ion irradiation. By using a helium ion microscope (HIM), we are able to reduce the width of exposure area down to $5 \mathrm{~nm}$. Sourcedrain conductance of side-gated GNR has been measured and the gate capacitances were extracted.
\end{abstract}

\section{INTRODUCTION}

Graphene quantum dot (GQD) is a potential platform to realize spin qubit owing to predicted long coherence time in graphene[1]. However, several issues prevent the effective confinement in GQD. The absence of a band-gap in graphene poses challenge to completely shut off the current. In addition, electrons in graphene are Dirac Fermions with a linear dispersion relation associated with the well-known Klein paradox, in which electrons could tunnel into a potential barrier with no reflection. This unique feature makes electrostatically defined potential barriers not feasible for monolayer graphene.

One method to create a transport gap in graphene is to lithographically reduce the channel size beyond the active width of GNR of $\sim 16 \mathrm{~nm}$. This method has been used to define constrictions in various GQD structures [2]. However further downscaling requires advanced patterning technology beyond the resolution of electron beam lithography (EBL). Recent progress on direct milling using ion beam[3] has shown very high patterning resolution. This method facing the problems of process integration and damage issue from high energy bombardment of substrates[4]. Here, we propose a new fabrication technique that tackles these issues by combining EBL and modest ion irradiation in HIM.

\section{RESULTS AND DISCUSSION}

\section{A. Fabrication methods}

Figure 1 shows the schematic of our device. The GNR was prepared by etching the EBL-defined pattern into exfoliated graphene. Both channel width and gateto-channel distance are $50 \mathrm{~nm}$. The rest of the ribbon is $400 \mathrm{~nm}$ wide. Tunnel barriers were formed by irradiating $5 \mathrm{~nm}$ strips on the GNR by $\mathrm{He}^{+}$ions with an equivalent defect density of $\sim 8 \times 10^{12} \mathrm{~cm}^{-2}$ as estimated from Raman spectrum[4]. By patterning two barriers with close proximity, a quantum dot structure can be effectively created. Two side gates (SG) are responsible for controlling the potential in the barriers one plunger gate (PG) tunes the central island. It should be noted that when zooming at high magnification $(\sim 80,000)$, imaging dose is comparable with patterning dose. Therefore it is crucial to mask the GNR to avoid any undesired exposure.

\section{B. Preliminary measurement}

We measured room temperature (RT) conductance after one $5 \mathrm{~nm}$ strip was irradiated in the GNR channel (see Fig. 2). Figures 3, 4 show the contour plot of conductance as a function of $\mathrm{V}_{\mathrm{BG}}$ and $\mathrm{V}_{\mathrm{SG}}$ (Fig. 3) and $\mathrm{V}_{\mathrm{BG}}$ and $\mathrm{V}_{\mathrm{PG}}$ (Fig. 4), respectively. In the former case, two side gates are connected, i.e. $\mathrm{V}_{\mathrm{SG}(\mathrm{L})}=\mathrm{V}_{\mathrm{SG}(\mathrm{R}) \text {. Two }}$ distinct slopes (the trajectory of charge neutrality point (CNP)) can be observed, from which the gate capacitance can be observed outlining four different potential configurations: $p-n, p-p, n-n$ and $n-p$. The side gate capacitances can be estimated from the diagonal slope (thick dashed line), $C_{S G}=C_{B G}\left(\frac{\Delta V_{S G}}{\Delta V_{B G}}\right)^{-1} \approx$ $72 n F$.Similarly, $C_{S G} \approx 60 n F$. Another notable feature is the unipolar transport at $p$ - $n$ regime. However, the role of irradiation played here is yet clear at this point. Higher dose may be needed for more observable change.

\section{CONCLUSION}

$5 \mathrm{~nm}$ wide strip was irradiated on a side-gated GNR. Room temperature conductance measurement showed electrostatic modulation on the channel by side gates. These can be used as control gates to tune the potentials in both barriers and quantum dots formed by irradiation. This work was partly supported by JAPAN Grant-inAid for Scientific Research (S) 25220904.

\section{REFERENCES}

[1] B. Trauzettel, D. V. Bulaev, D. Loss, and G. Burkard, "Spin qubits in graphene quantum dots," Nat. Phys., vol. 3, no. 3, pp. 192-196, Feb. 2007.

[2] L. A. Ponomarenko, F. Schedin, M. I. Katsnelson, R. Yang, E. W. Hill, K. S. Novoselov, and A. K. Geim, "Chaotic Dirac billiard in graphene quantum dots.," Science, vol. 320, no. 5874, pp. 356-8, 2008.

[3] N. Kalhor, S. A. Boden, and H. Mizuta, "Sub-10 $\mathrm{nm}$ patterning by focused $\mathrm{He}$-ion beam milling for fabrication of downscaled graphene nano devices," Microelectron. Eng., vol. 114, pp. 70-77, 2014. 
[4] S. Hang, Z. Moktadir, and H. Mizuta, "Raman study of damage extent in graphene nanostructures

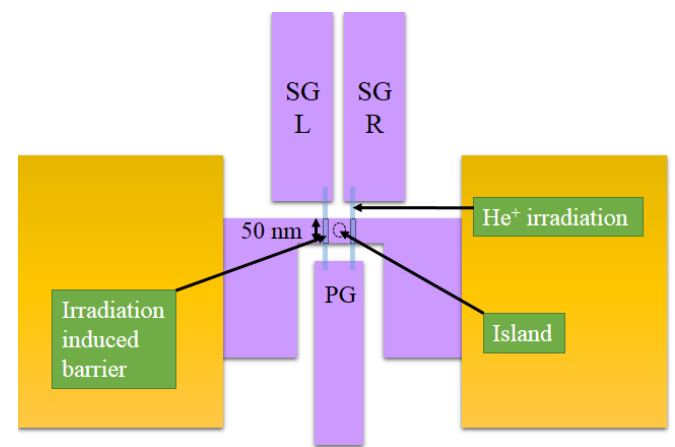

Fig.1 Schematics of our device. Single quantum dot is confined by two strips (blue) of insulating area made by ion irradiation.

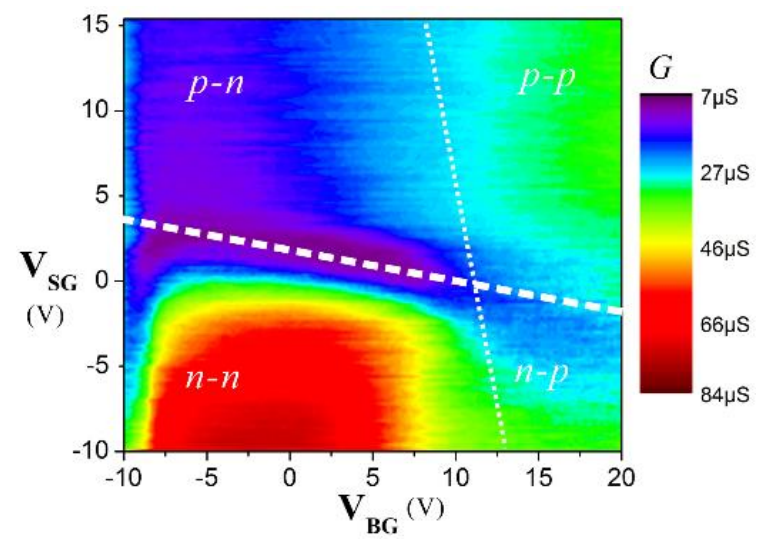

Fig.3 Contour plot of the source-drain conductance as a function of $\mathrm{V}_{\mathrm{SG}}$ and $\mathrm{V}_{\mathrm{BG}}$. White dashed lines indicate CNPs for the bare (thin) and SG-controlled (thick) region

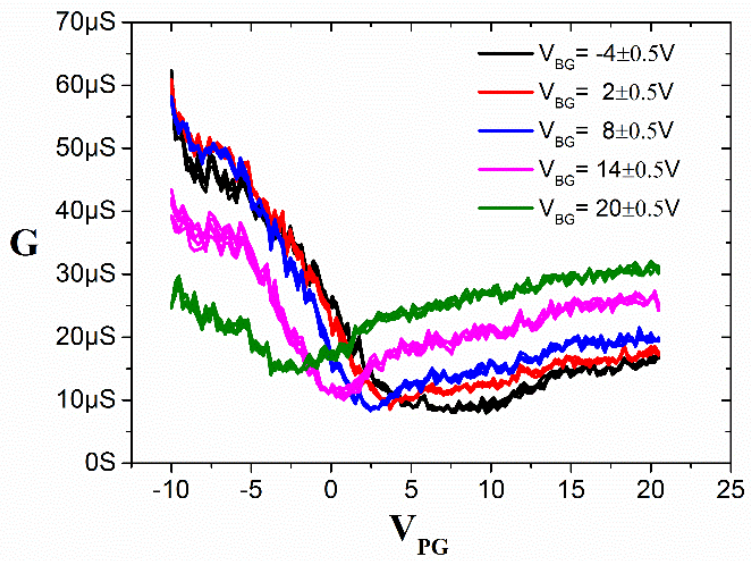

Fig. 5 Conductance as a function of $\mathrm{V}_{\mathrm{SG}}$ for $\mathrm{V}_{\mathrm{BG}}=-8,-4,0,4$ and $8 \mathrm{~V}$, as extracted from Fig.3. Unipolar transport can be observed by the black line. carved by high energy helium ion beam," Carbon, vol. 72, pp. 233-241, 2014.

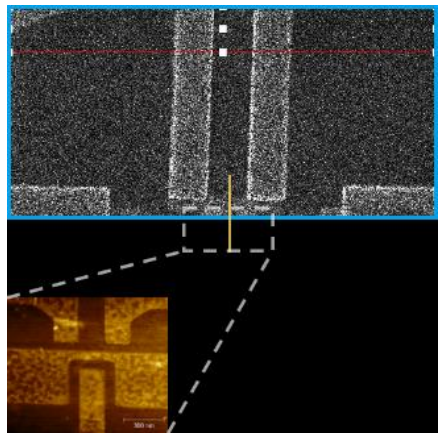

Fig.2 HIM irradiation of $5 \mathrm{~nm}$ strip in the channel (yellow vertical line). The blue frame outlines the image region. Inset shows the AFM image of our device.Scale bar is $300 \mathrm{~nm}$.

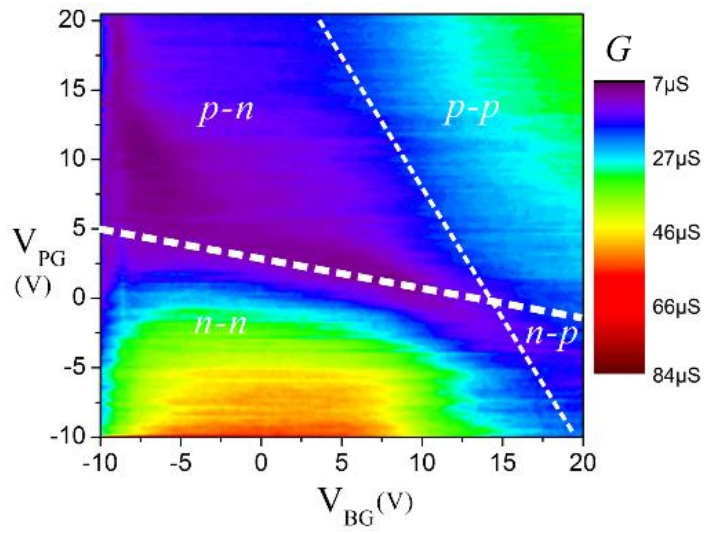

Fig.4 Contour plot of the source-drain conductance as a function of $V_{\mathrm{SG}}$ and $\mathrm{V}_{\mathrm{BG}}$. White dashed lines indicate the CNPs for the bare (thin) and PG-controlled (thick) regions.

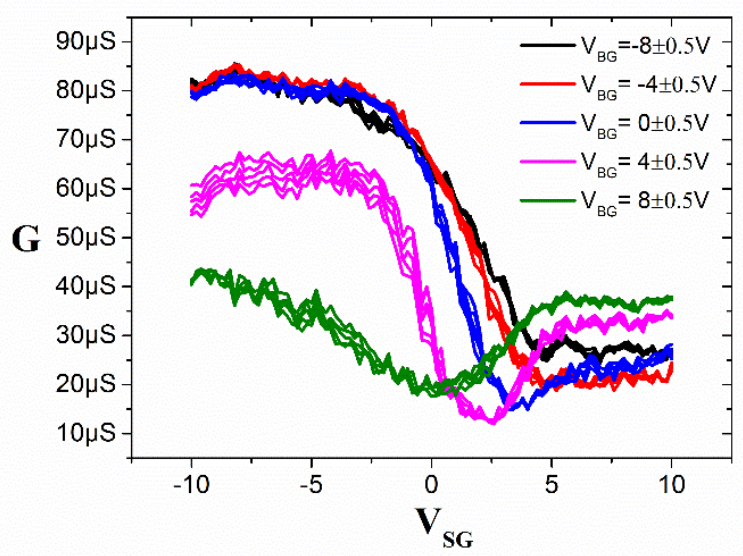

Fig. 6 Conductance as a function of $\mathrm{V}_{\mathrm{SG}}$ for $\mathrm{V}_{\mathrm{BG}}=-$ $4,2,8,14$ and $20 \mathrm{~V}$, as extracted from Fig.4. 